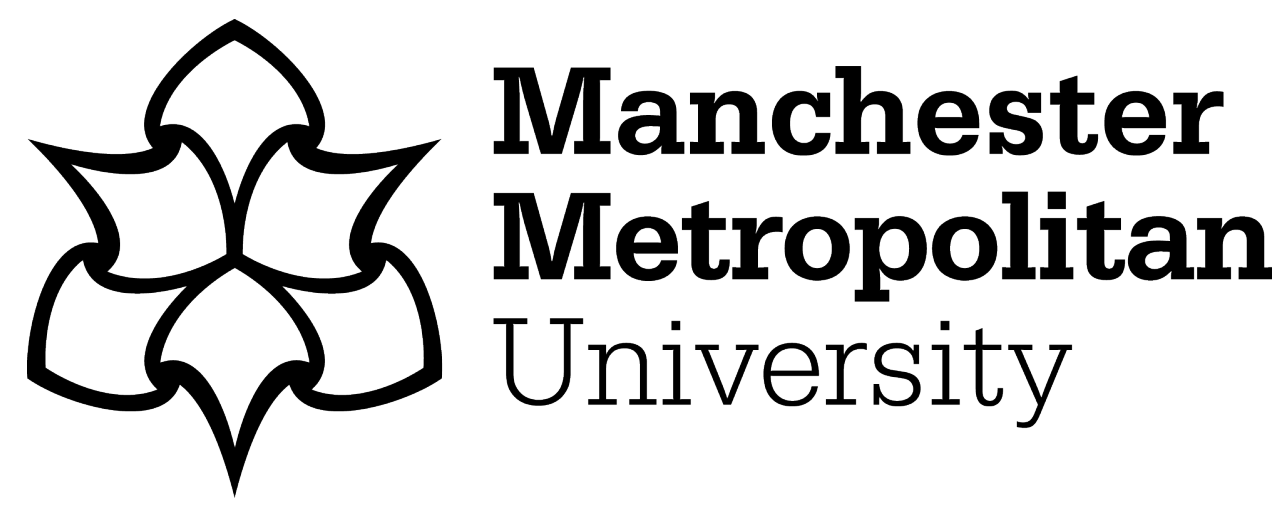

Opdebeeck, C ORCID logoORCID: https://orcid.org/0000-0003-0402-0984 (2020) Self-reported memory and depression over time. International Psychogeriatrics, 32 (6). pp. 681-683. ISSN 1041-6102

Downloaded from: https://e-space.mmu.ac.uk/627393/

Version: Accepted Version

DOI: https://doi.org/10.1017/S1041610219001923

Please cite the published version 


\section{Self-reported memory and depression over time}

Self-reported cognitive difficulties or decline have been frequently associated with an increased risk of developing dementia (Jessen et al., 2014; Reisberg et al., 2010). However, other studies have noted no association between self-reported cognitive difficulties and decline and subsequent dementia (Jorm et al., 1997). There has also been significant debate as to the associations between self-reported cognitive difficulties and depression. Specifically, as to whether depression results in people reporting more cognitive difficulties or if people experience an increase in objective cognitive difficulties due to depression (e.g. Yates et al., 2015; Zlatar et al., 2014). The paper by Hill and colleagues (2019), investigating the temporal bi-directional associations between self-reported memory and depressive symptoms, covers two of the important potential issues in investigating these associations, the differing methods used in measurement of self-reported cognitive difficulties and the temporal association with depressive symptoms. By addressing the associations of self-reported memory with depressive symptoms overtime, they are able to highlight the importance of considering how self-reported cognition is measured as well as its complex association with depression. These are key considerations that need to be addressed if self-reported cognition is going to be a useful tool in predicting future cognitive decline or dementia.

Many different approaches have been used to assess self-reported cognitive difficulties, so much so that even the terminology used can be somewhat controversial. The most common cognitive domain considered is memory but even within this single domain, there are wide variations in how self-reported memory is assessed. These methods range from single items to complex multiquestion measures and from asking people to report on their current memory, to compare themselves to others or to reflect on changes over specific time periods (Rabin et al., 2015). These variations may go some way in explaining the disparities in findings. Hill and colleagues address this issue by including three methods of assessing subjective memory - frequency of memory problems, perceived 1-year decline, and perceived 10-year decline. This allowed them to investigate the differences in methods of assessing self-reported memory in relation to depressive symptoms. Crosssectional studies have noted that the measurement of self-reported cognitive difficulties can influence whether they are associated with mood and with cognitive function. Opdebeeck and colleagues (2019) reported different associations of self-reported cognitive difficulties with objective cognition and mood by style of question asked and by cognitive domain, with more consistent associations of self-reported cognitive difficulties with mood than with cognition. The study by Opdebeeck and colleagues was cross-sectional so while it could highlight the importance of considering the method of assessing self-reported cognitive difficulties, it could not provide any information on the temporal associations or directionality.

A key issue in definitively identifying whether selfreported cognitive difficulties are to prove a useful predictor of future risk of dementia is in detangling the complex associations between self-reported cognition and depression. The study by Hill and colleagues set out to address this issue using data from the Einstein Aging Study, a longitudinal cohort study of community-dwelling people aged over 70 . The study included 1,163 participants who had no clinical diagnosis of mild cognitive impairment or dementia during the study. There were up to 11 waves of data for each participant, and the sample represented a relatively diverse population. As mentioned above, self-reported memory was assessed in three different ways, while depressive symptoms were assessed with the commonly used 15-item Geriatric Depression Scale (GDS-15; Sheikh \& Yesavage, 1986). The total GDS was calculated with 14 questions with the item "Do you feel you have more memory problems than most?" excluded to avoid conflating self-reported memory with depressive symptoms. Multilevel linear modeling adjusted for age, sex, education, race, and income was used to assess the temporal associations between the three measures of self-reported memory and depressive symptoms. There were medium associations between the three self-report memory items at baseline, indicating that while they are certainly related, these measures likely taped into different aspects of self-reported memory. At baseline, participants who reported higher frequency of memory problems or memory decline in comparison to 1 and 10 years ago reported higher levels of depressive symptoms than those who did not report memory decline (Hill et al., 2019). 
In relation to the key temporal relationships between self-reported memory and depressive symptoms, Hill and colleagues report that in their sample the frequency of self-reported memory problems at any particular wave did not predict levels of depressive symptoms at subsequent waves. Similarly, depressive symptoms did not predict frequency of memory problems at subsequent waves. Additionally, self-reported 1-year decline did not predict subsequent depressive symptoms nor did depressive symptoms predict subsequent reports of 1-year decline. Reports of 10-year decline did not predict future depressive symptoms; however, depressive symptoms did predict future reports of 10-year memory decline. The other important finding is that while no self-report memory assessment predicted future depressive symptoms, both self-report memory decline questions co-varied with depressive symptoms, in that increases in selfreported memory decline were associated with increased depressive symptoms at each of the time points. While depressive symptoms predicted concurrent changes in self-reported memory only in those who were older at baseline, these findings potentially indicate important considerations into the consistency of associations between self-reported memory and depressive symptoms that need to be taken into account.

The study by Hill and colleagues adds growing weight to the argument that consistency in how selfreported cognitive difficulties or changes are measured is needed. As noted, reviews have identified huge variation in the methods used across studies to assess self-reported cognition (e.g. Hill et al, 2016; Rabin et al., 2015). Attempts are being made to develop a standardized approach, for example, by the Subjective Cognitive Decline Initiative; however, there is still a lot of inconsistency within studies. While Hill and colleagues study does provide some insight into how different approaches to assessing self-reported memory may influence the level at which difficulties or changes are endorsed and their associations with depressive symptoms, there are some limitations. Notably, each approach used a single question and only assessed subjective memory. As noted by Rabin et al. (2015) and Opdebeeck et al. (2019), utilizing comprehensive assessments of subjective cognition and assessing different domains may also influence levels of endorsement and associations with other variables. Another important consideration is the low levels of depressive symptoms in this sample. As Hill and colleagues rightly note, low levels of depressive symptoms are to expected in a sample of communitydwelling older people; however, it does still mean that it is not possible to consider whether clinically significant depression may be a key factor. This could be important, as it is likely that clinically significant depression may have a greater impact both on perception of cognition and on actual cognition.

Theories such as the Hopelessness Theory of Depression (Abramson et al., 1989) may better explain the associations between clinical levels of depression and subjective appraisals of functioning than the associations with low-level symptoms. Depression, especially at clinical levels, has frequently been identified as a risk factor or marker for current poorer cognitive function and future decline and dementia (Almeida et al., 2017; Deckers et al., 2015; Opdebeeck et al., 2018). This link between depression and cognitive function may be due to some shared underlying mechanism (e.g. Byers and Yaffe, 2011). These potential associations could add a layer of complication into the associations between subjective memory difficulties or decline and depressive symptoms that are not considered by Hill and colleagues.

While there may be some limitations to Hill and colleagues study, which are clearly noted by the authors within the study, the strengths should be applauded. The study utilizes data from a large cohort of people aged over 70 and represents a relatively diverse sample, in terms of income at least, with a significant follow-up period. It highlights that there are important questions that can be addressed via secondary data analysis of large cohort study data. While there are limitations to this approach, notably in the often limited questions available on more specific topics, the availability of large data sets to address questions of note should be considered by more researchers. There is a wealth of longitudinal cohort data available to researchers, which can be used to address a wide variety of topics once the correct permissions have been granted. However, it is important to note that as with the study under consideration here, this can lead to limitations, for example, in the scope of measurement of selfreported memory difficulties and decline. Further research specifically designed to address this topic is still needed to disentangle the complicated associations between self-reported cognitive difficulties or decline and depression and future cognitive decline or dementia risk.

In sum, the study by Hill and colleagues is a valuable addition to the research on the complicated associations of self-reported memory difficulties and decline over 1-year and 10-year periods with depressive symptoms. It highlights that the associations can differ by the method used to assess self-reported memory and provide an insight into the variations in the associations over time. While there was no evidence to support that self-reported memory can 
predict future depressive symptoms a year later, the consistency of the covariance in self-reported memory and depressive symptoms over time highlights the importance of considering this association in clinical and research settings.

\section{Carol Opdebeeck}

Department of Psychology, Manchester Metropolitan University, Manchester, M15 6GX, UK

Email: c.opdebeeck@mmu.ac.uk

\section{References}

Abramson, L. Y., Metalsky, G. I. and Alloy, L. B. (1989). Hopelessness depression: a theory based subtype of depression. Psychological Review, 96, 358.

Almeida, O. P., Hankey, G. J., Yeap, B. B., Golledge, J. and Flicker, L. (2017). Depression as a modifiable factor to decrease the risk of dementia. Translational Psychiatry, 7, e1117. doi: $10.1038 /$ tp. 2017.90

Byers, A. L. and Yaffe, K. (2011). Depression and risk of developing dementia. Nature Reviews Neurology, 7, 323-331. doi: 10.1038/nrneurol.2011.60

Deckers, K. et al. (2015). Target risk factors for dementia prevention: a systematic review and Delphi consensus study on the evidence from observational studies. International fournal of Geriatric Psychiatry, 30, 234-246. doi: 10.1002/gps.4245

Hill, N. L. et al. (2016). Subjective cognitive impairment and affective symptoms: a systematic review. The Gerontologist, 56, e109-e127. doi: 10.1093/geront/gnw091

Hill, N. L. et al. (2019). Longitudinal relationships among depressive symptoms and three types of memory self-report in cognitively intact older adults. International Psychogeriatrics, 32, 719-732. doi: 10.1017/ S104161021900084X

Jessen, F. et al. (2014). A conceptual framework for research on subjective cognitive decline in preclinical Alzheimer's disease. Alzheimer's E Dementia, 10, 844-852. doi: 10. 1016/j.jalz.2014.01.001
Jorm, A. F., Christensen, H. E. K. A., Korten, A. E., Henderson, A. S., Jacomb, P. A. and Mackinnon, A. (1997). Do cognitive complaints either predict future cognitive decline or reflect past cognitive decline? A longitudinal study of an elderly community sample. Psychological Medicine, 27, 91-98. doi: 10.1017/ S0033291796003923

Opdebeeck, C., Matthews, F. E., Wu, Y. T., Woods, R. T., Brayne, C. and Clare, L. (2018). Cognitive reserve as a moderator of the negative association between mood and cognition: evidence from a population-representative cohort. Psychological Medicine, 48, 61-71. doi: 10.1017/ S003329171700126X

Opdebeeck, C., Yates, J. A., Kudlicka, A. and Martyr, A. (2019). What are subjective cognitive difficulties and do they matter? Age and Ageing, 48, 122-127. doi: 10.1093/ageing/ afy 148

Rabin, L. A. et al. (2015). Subjective cognitive decline in older adults: an overview of self-report measures used across 19 international research studies. Fournal of Alzheimer's Disease, 48, S63-S86. doi: 10.3233/JAD-150154

Reisberg, B., Shulman, M. B., Torossian, C., Leng, L. and Zhu, W. (2010). Outcome over seven years of healthy adults with and without subjective cognitive impairment. Alzheimer's E Dementia, 6, 11-24. doi: 10.1016/j.jalz. 2009.10.002

Sheikh, J. I. and Yesavage, J. A. (1986). Geriatric Depression Scale (GDS): recent evidence and development of a shorter version. Clinical Gerontologist: The fournal of Aging and Mental Health, 5, 165-173. doi: 10.1300/J018v05n01_09

Yates, J. A., Clare, L., Woods, R. T. and Matthews, F. E. (2015). Subjective memory complaints are involved in the relationship between mood and mild cognitive impairment. Fournal of Alzheimer's Disease, 48, S115-S123. doi: 10.3233/JAD-150371

Zlatar, Z. Z., Moore, R. C., Palmer, B. W., Thompson, W. K. and Jeste, D. V. (2014). Cognitive complaints correlate with depression rather than concurrent objective cognitive impairment in the successful aging evaluation baseline sample. Fournal of Geriatric Psychiatry and Neurology, 27, 181-187. doi: 10.1177/0891988714524628 